\title{
The Change Clinic Counselling Service: Blending Client-Centred and Strength-Based Practices to Improve Mental Health Services to Families
}

\author{
Stephen Ellenbogen, and Heather J. Hair \\ Memorial University \\ June Kirkland Smith \\ Eastern Health \\ Peter Wilton \\ Memorial University
}

\begin{abstract}
Operating in a small urban Canadian location, Change Clinic is a brief counselling service intended for child and adolescent mental health issues. It blends principles from different strength-based and clientcentred approaches to counselling (e.g., solution-focused, narrative, and single session therapies). Using the parent-report version of the Strengths and Difficulties Questionnaire, changes in child and adolescent mental health difficulties were assessed. Compared to pre-test scores, significant improvements in behavioural, emotional, and hyperactivity problems were observed six months after delivery of the service. Effect size was estimated to be moderate (.62) based on the Added Value Score calculation. Almost three quarters of clients were served in one to two sessions.
\end{abstract}

Keywords: child and adolescent, mental health services, alternative therapies, quantitative evaluation

Stephen Ellenbogen, School of Social Work, Memorial University, St. John's, Newfoundland \& Labrador; Heather J. Hair, School of Social Work, Memorial University, St. John's, Newfoundland \& Labrador; June Kirkland Smith, Eastern Health, St. John's, Newfoundland \& Labrador; and Peter Wilton, Nova Scotia Health Authority, Halifax, Nova Scotia.

Peter Wilton is now at Hill + Knowlton Strategies, Ottawa, Ontario.

The study was supported by funding from the Janeway Foundation, Eastern Health Region, Newfoundland and Labrador. The authors declared that they have no competing or potential conflicts of interest.

Correspondence concerning this article should be addressed to Stephen Ellenbogen, Associate Professor, School of Social Work, Memorial University, P.O. Box 4200, St. John's, Newfoundland \& Labrador, Canada A1C 5S7. Email: sellenbogen@mun.ca 


\section{RÉSUMÉ}

Situé dans une petite ville canadienne, Change Clinic est un service de consultation de courte durée destinée aux enfants et aux adolescents aux prises avec des problèmes de santé mentale. Le traitement s'inspire des principes constitutifs de différentes approches d'aide et de soutien fondées sur les forces et le client (ex., les thérapies axées sur la recherche de solutions, les thérapies narratives et les thérapies à séance unique). À l'aide de la version à l'usage des parents du questionnaire portant sur les points forts et les difficultés (Strengths and Difficulties Questionnaire), une évaluation a été faite des changements relatifs aux problèmes de santé mentale des enfants et des adolescents. Six mois après la prestation des services, des améliorations importantes ont été observées en ce qui concerne les problèmes comportementaux, émotionnels et d'hyperactivité, et ce, en comparaison des résultats obtenus lors des tests préliminaires. Sur la base du calcul de la valeur ajoutée (Added Value Score), il a été déterminé que l'ampleur des changements était modérée $(0,62)$. Près des trois quarts des clients ont pu recevoir leur traitement en une ou deux séances.

Mots clés : enfants et adolescents, services de santé mentale, thérapies de substitution, évaluation quantitative

\section{INTRODUCTION}

Client-centred and strength-based (CCSB) practices represent a marked paradigm shift for child and adolescent mental health services (CAMHS). Instead of expert-driven medicalized processes that seek to diagnose and treat illness, the focus is on developing co-creative, collaborative relationships between service providers and clients to determine the dominant problem and discover and utilize clients' resources and resiliencies for solution planning. CCSB approaches accommodate families' need for brief and expedient services (Perkins, 2006). Also, therapeutic processes may be less stigmatizing because diagnoses (i.e., clinical labels) are not required; hence, families don't feel blamed for having difficulties (Goldberg \& Campbell, 1997; Lethem, 2002; Partridge, Redmond, Williams, Black, \& Richardson, 1999; Wheeler, 2001). A focus on manageable change typically reduces the number of sessions and provides helpful services according to participants. Some have suggested that CCSB approaches are ideally suited to resolving long recognized inadequacies of CAMHS in urban and rural settings, notably that those in need face barriers to accessing services, including long wait times and burdensome treatments (Church, Ellenbogen, \& Hudson, 2016; Hair, Shortall, \& Oldford, 2013; Heywood et al., 2003; Miller \& Slive, 2004; Stallard \& Sayers, 1998).

Effectiveness of CCSB approaches is confirmed in numerous evaluations of CAMHS services. Solutionfocused brief therapy (SFBT) was found to be associated with reductions in mental health symptoms, behaviour problems, and anxiety (Cepukiene \& Pakrosnis, 2011; Daki \& Savage, 2010; Franklin, Moore, \& Hopson, 2008; Moore, 2002; Seagram, 1997; Vostanis, Anderson, \& Window, 2006). Administratively, SFBT was found to integrate well with a public health network, and to fill previously unmet needs for local mental health services that do not require highly specialized care (Worrall-Davies, Cottrell, \& Benson, 2004). Single session SFBT was reported to be successful for treating mental health issues and improving global functioning (O'neill \& Rottem, 2012). Also, families using brief therapy and walk-in services have demonstrated greater improvements in mental health than clients of a treatment-as-usual service (Barwick 
et al., 2013). Qualitative findings generally point to CCSB practices as particularly suited for gaining user satisfaction and engagement (Hair et al., 2013).

In some evaluations however, the outcomes of CCSB services compared less favourably to those of alternative treatments (Yarbrough \& Thompson, 2002; Wilmshurst, 2002). Other evaluations revealed improvements in some mental health areas but not in others (Cepukiene \& Pakrosnis, 2011). Finally, there are anecdotal reports that CCSB approaches might be less suited to clients with severe difficulties or preference for traditional models of treatment (Allison, Roeger, Dadds, \& Martin, 2000; Stallard \& Sayers, 1998).

In sum, CCSB services have demonstrated mental health outcomes that are comparable to other service models, that is, they are modestly effective. Now that these practices have been validated, a next step might be to seek conceptual and administrative refinement. The latter refers to innovation in service delivery, such as offering a variety of like-minded treatments or bringing the service closer to those in need. Conceptualization refinement might involve blending techniques and principles from different CCSB approaches or adapting approaches to serve specific needs or populations. For example, a mixed solution-focused and empowerment model was found effective for trauma issues (Georgiades, 2008). The current study is a pre-experimental evaluation of another such practice. Using the Strengths and Difficulties Questionnaire (SDQ), parents reported on the mental health of their children just prior to treatment, and three months and six months afterward. An estimate of treatment effect was calculated using the added value score (Ford, Hutchings, Bywater, Goodman, \& Goodman, 2009) for the SDQ. This score is derived from an algorithm intended to approximate change in client scores after factoring out spurious effects such as regression to the mean.

\section{Change Clinic Counselling Service}

Change Clinic is a CCSB counselling approach that draws assumptions and skills from SFBT, narrative, single-session, and strength-based practices. Each session is approached by the service provider as if it was intended to be the only session; up to two additional single sessions are possible. The following questions are used to focus the conversation, "What one thing is most important to you right now? ... Given that, are you more interested in knowing what to do to change it or do you want a better or different understanding?" (Hair, et al., 2013, p.22). True to client-centred philosophy, a key objective of the service provider is to co-create a healing strategy with clients. Mirroring this spirit, Change Clinic was co-created by academic and local CAMHS partners. A notable output of this collaboration was a shared interest in evaluating the service. In an initial study, high levels of client satisfaction were reported and client narratives suggested that Change Clinic promotes communication, solution seeking, and hope within families (Hair et al., 2013).

Beginning in 2010, Change Clinic was offered one day a week as a counselling service for children, adolescents, and their families in an urban area of the province of Newfoundland \& Labrador, Canada. Except for those who were judged better served by another organization (e.g., child protective services, child psychiatry, addictions), intake coordinators advised potential clients that they could take the next available appointment for Change Clinic or a treatment-as-usual service. The service was explained, and approximate wait times for each service were provided. 


\section{METHODS}

\section{Ethics Approval}

Approval was obtained from the Memorial University Interdisciplinary Committee on Ethics in Human Research as well as from the Eastern Health Regional Research Proposal Approval Committee.

\section{Participants}

Families who attended the Change Clinic between June 2013 and April 2014 were invited to participate in the study. A total of 55 parents ( $81 \%$ of those approached) completed pre-test measures; $84 \%$ of reporting parents were mothers; $45 \%$ of participating children were boys, who were found to be younger $(\mathrm{M}=8.4 ; \mathrm{SD}=3.4)$ than girl participants $(\mathrm{M}=10.8 ; \mathrm{SD}=2.1, t=(32) 3.1, p=.04)$. There were no gender differences in pre-test SDQ total difficulties scores (males: $\mathrm{M}=14.9, \mathrm{SD}=6.5$; females: $\mathrm{M}=15.7, \mathrm{SD}=$ 5.9). Of families who participated at pre-test, 23 (42\%) completed a 3-month follow-up questionnaire, and 24 (44\%) completed a 6-month follow-up. The two most common reasons given for not participating were not having time and uncomfortable with participation.

\section{Measures}

The SDQ (Goodman, 2001) is a validated assessment of child/youth mental health and well-being that has been extensively used for research and clinical purposes. A total difficulties score is calculated as the sum of four subscales measuring conduct, emotional, hyperactivity and peer problems. A prosocial subscale is also included. Subscales contain five items to which respondents answer on a scale ranging from 0 (not true) to 2 (certainly true). Two additional items evaluate whether (1) child problems are much worse, a bit worse, about the same, a bit better, or much better since coming to clinic, and (2) clinic has helped in other ways: not at all, a little, a medium amount, or a great deal. A total impact score is calculated as the total score of four items pertaining to how much the problems affect dimensions of the child social world, also on a scale from 0 (not at all or a little) to 2 (a great deal). The authors provide "slightly raised" and "highsubstantial risk of clinically significant problems" thresholds for total difficulties and problem subscales (for further information, see www.sdqinfo.com/). We examined child- and parent-report data at pre-test and parent-report data at post-test.

\section{Service Amount Preference}

The number of treatment sessions that clients opted to initiate (range $=1-5$ ) was monitored.

\section{Analysis}

Only 16 families provided information at all three time points. To maximize statistical power, we conducted comparisons of the pre-test scores to 3- and 6-month post-test scores separately; hence pre-test scores for comparisons are not identical because they involve slightly different samples. Paired sample ttests, Repeated Measure General Linear Models, Related-Samples Wilcoxon Signed Rank Tests, and Chi 
Square were used for testing statistical significance. We used recently devised techniques for estimating of treatment effect (Ford et al., 2009), using the following formulae:

Raw SDQ Added Value Score (in SDQ points) $=2.3+0.8 *$ baseline total difficulties score $+0.2 *$ baseline impact score $-0.3 *$ baseline emotional difficulties subscale score - follow-up total difficulties score.

Raw change score (in SDQ points) = baseline total difficulties score - follow-up total difficulties score

\section{RESULTS}

\section{Comparisons of Study Completers and Non-Completers}

We found no significant differences between study completers and non-completers (i.e., families whose parents completed no follow-up questionnaire) in terms of child age, number of Change Clinic sessions, and parent-reported child difficulties at pre-test. Parents were more likely to complete post-tests if their children were male $(72 \%)$ than female $(47 \%)$, but the difference only neared significance, $\chi(1, N=54)=$ $3.6, p=.052$. Based on self-report SDQ scores at pre-test, youth from non-completing families reported higher scores for total difficulties $(\mathrm{M}=21.8, \mathrm{SD}=5.4)$, emotional problems $(\mathrm{M}=7.3, \mathrm{SD}=1.4)$, and peer problems $(\mathrm{M}=4.1, \mathrm{SD}=2.6)$, as compared to youth from completing families (total difficulties: $\mathrm{M}=14.1$, $\mathrm{SD}=4.5, t=(16) 3.1, p=.009$; emotional: $\mathrm{M}=5.2, \mathrm{SD}=2.1, t=(16) 2.2, \mathrm{p}=.05$; peer: $\mathrm{M}=1.9, \mathrm{SD}=$ $1.7, t=(16) 2.1, p=.05)$. Finally, no significant differences were found between parent-report and youth self-report SDQ scores at pre-test.

\section{Change in Mental Health Issues}

We found significant reductions in parent-reported SDQ scores for total difficulties, emotional problems, conduct problems at both the 3- and 6-month follow-ups and for Hyperactivity at 6 months only (Table 1, next page). The raw change score for SDQ total difficulties for the pre- to 6-month comparison was .89 (C.I., .55 to 1.22). Based on a calculation of the Added Value Score, which factors out non-treatment effects that might contribute to positive change in psychopathology scores, the estimated effect size for the intervention was .62 (C.I., 22 to 1.03 ).

Although mean differences were largest for emotional problems, a different trend emerged from exploring clinically significant change. Based on the SDQ Interpretation guide (Goodman, 2001; p. 17), 14, 8 , and 8 children respectively were rated as being at substantial risk of clinically significant emotional, conduct, and hyperactivity problems at pre-test. By 6 months, $43 \%, 38 \%, 63 \%$ of these children, respectively, were no longer rated as such. Unfortunately, statistical analyses of these improvements were not possible. Compared to the pre-test, a smaller number of clinical problems were noted at 3 months (pre $\mathrm{M}=1.4, \mathrm{SD}=$ 1.0; post $\mathrm{M}=0.6, \mathrm{SD}=0.9$ ) and at 6 months (pre $\mathrm{M}=1.5, \mathrm{SD}=1.2$; post $\mathrm{M}=1.0, \mathrm{SD}=1.3$ ), however, only the decrease at the 3-month mark was statistically significant, (Wilcoxon signed-ranks test) $Z=19.5, p<0.05$.

At three months, a large majority of parents felt that coming to the Change Clinic had helped with their children's problems (48\% responding much better, $48 \%$ a little better, and $4 \%$ about the same, and $0 \%$ worse) and in other ways (26\% indicating a great deal, $52 \%$ a medium amount, $22 \%$ a little, $0 \%$ not at all). There was some indication of a polarizing of views (i.e., some more positive and others less so) at six 
Table 1

T-Test Comparisons of SDQ Parent Reports on Child/Youth Problems: Pre-Test to 3-Month and Pre-Test to 6-Month Following Change Clinic

\begin{tabular}{|c|c|c|c|c|c|c|}
\hline & \multicolumn{2}{|c|}{$\begin{array}{l}\text { Parent-Reported } \\
\text { Mean Scores (SD) }\end{array}$} & \multicolumn{4}{|c|}{$\begin{array}{l}\text { Parent-Reported } \\
\text { Mean Scores (SD) }\end{array}$} \\
\hline & Pre-test & 3 Months & $t$ & Pre-test & 6 Months & $t$ \\
\hline \multicolumn{7}{|l|}{ SDQ } \\
\hline Total difficulties ${ }^{\mathrm{a}}$ & $15.2(5.4)$ & $11.4(5.1)$ & $3.6^{* *}$ & $15.8(6.2)$ & $10.6(7.6)$ & $5.4^{* *}$ \\
\hline Emotional problem & $5.4(2.7)$ & $3.6(2.4)$ & $4.2^{* *}$ & $5.2(3.2)$ & $3.3(2.8)$ & $4.4^{* *}$ \\
\hline Conduct problem & $2.3(1.8)$ & $1.5(1.3)$ & $2.3^{*}$ & $2.8(1.9)$ & $1.8(2.0)$ & $3.2 * *$ \\
\hline Hyperactivity & $5.4(2.5)$ & $4.5(2.4)$ & 1.8 & $5.7(2.3)$ & $3.8(2.7)$ & $3.7 * *$ \\
\hline Peer problem & $2.0(1.9)$ & $1.9(1.9)$ & 0.5 & $2.1(2.0)$ & $1.8(1.9)$ & 1.5 \\
\hline Prosocial & $8.4(1.5)$ & $8.9(1.3)$ & -1.9 & $8.2(1.7)$ & $8.5(1.8)$ & -0.8 \\
\hline
\end{tabular}

a Sum or the four following subscales; For 3-month follow-up $\mathrm{N}=23$, for 6 months $\mathrm{N}=24$.

$* p<.05 * * p<.005$

months: $54 \%$ reported much better, $25 \%$ a little better, $13 \%$ about the same, and $8 \%$ worse for the item on helping child problems; and 33\% reported a great deal, $46 \%$ a medium amount, $17 \%$ a little, and $4 \%$ not at all for the item on helping in other ways.

\section{Service Amount Preference and Effect on Change in Mental Health Issues}

Amount of service preference was available for all 55 clients. Roughly half (49\%) opted for one session, $24 \%$ attended two sessions, and $16 \%$ attended three. Although practice protocols specify a maximum of three sessions, $9 \%$ received a fourth session and $2 \%$ received a fifth.

We examined whether dosage (i.e., number of Change Clinic sessions attended) would moderate the magnitude of change in SDQ Total Difficulties Scores from pre- to 3- and 6-month post-test. Based on the results of 2 x 2 time by dosage (1-2 sessions vs. 3+ sessions) Repeated Measure General Linear Models, there were no significant main effects for dosage or interaction effects. However, there was a slight trend toward an interaction effect, with greater improvement at six months for families receiving a high dose (M $(\mathrm{T} 2-\mathrm{T} 1)=7.1)$ as compared to those receiving a low dose $(\mathrm{M}(\mathrm{T} 2-\mathrm{T} 1)=4.6 ; F(22)=1.6, p=.22)$.

\section{DISCUSSION}

The results of this pre-experimental evaluation of Change Clinic are particularly promising. Improvements were noted in three of the most commonly diagnosed issues facing young people and their families (emotional, behavioural, and hyperactivity problems), thus supporting the application of this 
alternative counselling approach. Moreover, the estimated impact on child mental health is noteworthy. In a meta-analysis of 22 SFBT evaluations (Kim, 2008), effect sizes ranged from -.46 to 1.18; the added value score found for this study (.62) would rank third on this list. It must be cautioned, however, that treatment effects can't be inferred because of the lack of a control condition.

Contrasting the mean SDQ difficulties scores at pre-test to those at the 3-and 6-month follow-ups, we saw no evidence of re-emerging problems between the two follow-up points. If anything, the final follow-up yielded more positive results in that hyperactivity improvements were noted at six months only. However, the percentage of respondents who reported no improvement or worsening symptoms rose from $4 \%$ to $21 \%$ between three and six months. Further research is needed to better understand different trajectories following treatment.

In addition to the lack of a comparison group, a main limitation of this study is high attrition at follow-up periods; dropout of unsatisfied clients can inflate treatment effects. Also, the study involved a small sample from a single treatment site and relied on parent-reports. Hence, the results don't permit definitive conclusions, but rather serve as support for investing in further evaluation, using multiple outcome measures, control groups, and strategies for reducing attrition. Nevertheless, the evidence of this study provides encouraging support for the provision of time-sensitive, client-centred and strength-based counselling services with children, adolescents, and their families.

\section{REFERENCES}

Allison, S., Roeger, L., Dadds, V., \& Martin, G. (2000). Brief therapy for children's mental health problems: Outcomes in a rural setting. Australian Journal of Rural Health, 8, 161-166.

Barwick, M., Urajnik, D., Sumner, L., Cohen, S., Reid, G., Engel, K., \& Moore, J. E. (2013). Profiles and service utilization for children accessing a mental health walk-in clinic versus usual care. Journal of Evidence-Based Social Work, 10, 338-352.

Cepukiene, V., \& Pakrosnis, R. (2011). The outcome of solution-focused brief therapy among foster care adolescents: The changes of behavior and perceived somatic and cognitive difficulties. Children and Youth Services Review, 33, 791-797.

Church, M. J., Ellenbogen, S., \& Hudson, A. (2016). Falling through the cracks: An exploratory study of the perceived barriers to mental health services for rural and urban Cape Breton youth. Unpublished manuscript.

Daki, J., \& Savage, R. (2010). Solution-focused brief therapy: Impacts on academic and emotional difficulties. The Journal of Educational Research, 103, 309-326.

Ford, T., Hutchings, J., Bywater, T., Goodman, A., \& Goodman, R. (2009). Strengths and Difficulties Questionnaire AVSs: Evaluating effectiveness in child mental health interventions. The British Journal of Psychiatry, 194, 552-558.

Franklin, C., Moore, K., \& Hopson, L. (2008). Effectiveness of solution focused brief therapy in a school setting. Children and Schools, 30, 15-26.

Georgiades, S. D. (2008). A solution-focused intervention with a youth in a domestic violence situation: Longitudinal evidence. Contemporary Family Therapy, 30, 141-151.

Goldberg, D., \& Campbell, F. (1997). Empowerment: A three-session intervention. Child Psychology \& Psychiatry Review, 2, 34-37.

Goodman, R. (2001). Psychometric properties of the Strengths and Difficulties Questionnaire (SDQ). Journal of the American Academy of Child and Adolescent Psychiatry, 40, 1337-1345.

Hair, H. J., Shortall, R., \& Oldford, J. (2013). Where's help when we need it? Developing responsive and effective brief counseling services for children, adolescents, and their families. Social Work in Mental Health, 11, 16-33. 
Heywood, S., Stancombe, J., Street, E., Mittler, H., Dunn, C., \& Kroll, L. (2003). A brief consultation and advisory approach for use in child and adolescent mental health services: A pilot study. Clinical Child Psychology and Psychiatry, 8, 503-512.

Kim, J. S. (2008). Examining the effectiveness of solution-focused brief therapy: A meta-analysis. Research on Social Work Practice, 18, 107-116.

Lethem, J. (2002). Brief solution focused therapy. Child and Adolescent Mental Health, 7, $189-192$.

Miller, J. K., \& Slive, A. (2004). Breaking down the barriers to clinical service delivery: Walk-in family therapy. Journal of Marital and Family Therapy, 30, 95-103.

Moore, K. (2002). The effectiveness of solution-focused therapy on students with school-related behavioural problems. Austin: The University of Texas.

O’neill, I., \& Rottem, N. (2012). Reflections and leaming from an agency-wide implementation of single session work in family therapy. The Ausrtralian and New Zealand Journal of Family Therapy, 33, 70-83.

Partridge, I., Redmond, C., Williams, C., Black, J., \& Richardson, G. (1999). Evaluating family therapy in a child and adolescent mental health service. Psychiatric Bulletin, 23, 531-533.

Perkins, R. (2006). The effectiveness of one session of therapy using a single-session therapy approach for children and adolescents with mental health problems. Psychology \& Psychotherapy: Theory, Research \& Practice, 79, 215-227.

Seagram, B. C. (1997). The efficacy of solution-focused therapy with young offenders. Unpublished doctoral dissertation, York University, North York, Ontario.

Stallard, P., \& Sayers, J. (1998). An opt-in appointment system and brief therapy: Perspectives on a waiting list initiative. Clinical Child Psychology and Psychiatry, 3, 199-212.

Vostanis, P., Anderson, L., \& Window, S. (2006). Evaluation of a family support service: Short-term outcome. Clinical Child Psychology and Psychiatry, 11, 513-528.

Wheeler, J. (2001). A helping hand: Solution-focused brief therapy and child and adolescent mental health. Clinical Child Psychology and Psychiatry, 6, 293-306.

Wilmshurst, L. A. (2002). Treatment programs for youth with emotional and behavioral disorders: An outcome study of two alternate approaches. Mental Health Services Research, 4, 85-96.

Worrall-Davies, A., Cottrell, D., \& Benson, E. (2004). Evaluation of an early intervention Tier 2 child and adolescent mental health service. Health \& Social Care in the Community, 12, 119-125.

Yarbrough, J. L., \& Thompson, C. L. (2002). Using single-participant research to assess counseling approaches on children's off-task behavior. Professional School Counseling, 5, 308-314. 